\title{
Real-Time Microfluidics-Magnetic Tweezers connects conformational stiffness with energy landscape by a single experiment
}

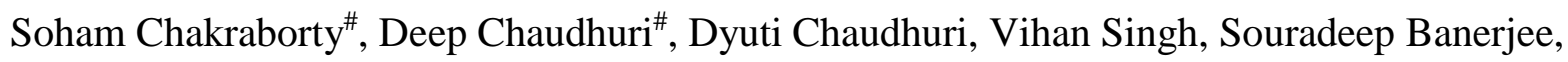
Shubhasis Haldar*

Department of Biological Sciences, Ashoka University, Sonepat, Haryana 131029

\# contributed equally to this work

*To whom correspondence may be addressed. Email- shubhasis.haldar@ashoka.edu.in 


\begin{abstract}
:
Studies of free energy, kinetics or elasticity are common to most disciplines of science. Detailed quantification of these properties demands number of specialized technologies. Furthermore, monitoring 'perturbation' in any of these properties, in presence of external stimuli (protein/DNA/drugs/nanoparticles etc.), requires multiple experiments. However, none of these available technologies can monitor these perturbations simultaneously in real time on the very same molecule in a single shot experiment.

Here we present real-time microfluidics-magnetic tweezers technology with the unique advantage of tracking a single protein dynamics for hours, in absence of any significant drift, with the flexibility of changing physical environment in real time. Remarkable stability of this technique allows us to quantify five molecular properties (unfolding kinetics, refolding kinetics, conformational change, chain flexibility, and $\Delta \mathrm{G}$ for folding/unfolding), and most importantly, their dynamic perturbation upon interacting with salt on the same protein molecule from a single experiment. We observe salt reshapes the energy landscape by two specific ways: increasing the refolding kinetics and decreasing the unfolding kinetics, which is characterized as mean first passage time. Importantly, from the same trajectory, we calculated the flexibility of the protein polymer, which changes with salt concentration and can be explained by our modified 'electrolyte FJC model'. The correlation between $\Delta \mathrm{G}$, kinetics and polymer elasticity strongly argues for a stiffness driven energy landscape of proteins. Having the advantage of sub nanometer resolution, this methodology will open new exciting window to study proteins - one such examples is demonstrated in this article: electrolyte driven conformational fluctuation under force, which was not studied before.
\end{abstract}




\section{Introduction:}

Each and every biomolecule, participating in biochemical reactions, undergoes continuous conformational fluctuations and functional dynamicity due to their kinetic and thermodynamic properties. Determination of the change in these properties during interaction, being the key drivers of energy landscape, determines the molecular mechanism of the reaction. There are different dedicated technologies available to quantify these changes: kinetics by stopped flow, thermodynamics by isothermal titration calorimetry or circular dichroism, conformational fluctuations by nuclear magnetic resonance or fluorescence correlation spectroscopy, polymer properties by atomic force microscopy, optical tweezers or magnetic tweezers ${ }^{1-10}$. However, none of them ever reports all these changes simultaneously in a single shot experiment on the very same molecule. These challenges arise due to two major limitations: i) absence of a microfluidic flow system to introduce new solution during experiment and, ii) lack of an attachment principle for the target protein, which causes protein detachment, while flushing the system chamber with new solution containing interactor molecules. Additionally, the challenges arise from the inability to observe the subnanometer conformational changes of small biomolecules under force.

Intracellular ion homeostasis plays an indispensable role in maintaining protein conformation by constantly changing ionic environment and thus, directly influence folding. Ion dependent polymer properties of DNA and RNA are widely studied ${ }^{11-13}$; however, there is no report on polymer properties of proteins under force. Notably, cytoplasmic compartments such as, endoplasmic reticulum, Golgi apparatus or lysosome, involved in secretory pathway have a $\mathrm{pH}$ gradient due to the variation in ion compositions which not only affect the folding of the secreting proteins but also of their resident proteins. Furthermore, various multidomain and polymeric proteins in cytoplasm such as, talin, actin work under force and due to extended topology; they are exposed to different ion conditions. How the elasticity of these proteins change as a function of force and electrolyte concentrations is still not known. Though, experimental and theoretical studies suggest that solvent quality affects the compaction of protein polymer, its role in further structure formation to promote folding is highly debated ${ }^{14}$. Additionally, a proper model is lacking, which combines both kinetics and thermodynamics to polymer science in a single frame.

Here, we introduce Real-Time Microfluidic-Magnetic Tweezers (RT-MMT) that not only quantifies all the above-mentioned physical properties in tandem but also detects their changes under the influence of various external stimuli (like, DNA, protein, nanoparticle etc.) on the very same molecule in a single shot experiment in one frame. Our integrated approach, by combining the microfluidic flow system with covalently linked magnetic tweezers, allows us to change the solution multiple times during experiment while tracking a single protein molecule. In the current work, we report the effect of ion concentration on the folding landscape and polymer elasticity of B1 domain of protein L. Our result shows that salt tilts the energy landscape towards folding by stabilizing the folded state and destabilizing the unfolded state. For the first time, we report an ion dependent protein collapse under force and propose an electrolyte freely jointed chain (FJC) model 
to explain the conformational flexibility in respect to salt concentration. Finally, we successfully combine these results with our kinetics and thermodynamic related observations to illustrate a comprehensive picture of protein elasticity which will open a new gateway for studying folding. Interestingly the data, we observed from our single molecule analysis, are in strong agreement with the multi-experiment observation, claiming their place as a multiplexed biophysical technology to study a wide range of questions in biology.

\section{Results and Discussion:}

\section{Real-Time Microfluidics-Magnetic Tweezers technology to study protein dynamics:}

Real-Time Microfluidics-Magnetic Tweezers (RT-MMT) have the unique advantage of constant monitoring the folding dynamics of a single protein, while introducing external stimuli (proteins/DNA/drugs/nanoparticles etc.) in a single experiment (Fig. 1A). Additionally, it provides us an exceptional way to monitor the mechanical unfolding and folding transitions of single protein by applying a force-clamp technology. The experiments were performed on polyprotein construct of protein L B1 domain, which was immobilized on glass surfaces using HaloTag-based covalent chemistry and anchored to paramagnetic beads by biotin-streptavidin chemistry. A pair of permanent magnets, placed above the microfluidics chamber, can apply a passive force-clamp upto $120 \mathrm{pN}$ with sub-pN resolution by generating a magnetic field. The pulling force is calculated as a function of distance between magnet and the paramagnetic beads attached to protein and force magnitude is inversely proportional with the distance (Fig. 1B). Fig. 1B demonstrates a representative force-clamp trajectory performed by changing magnet position from $4 \mathrm{~mm}$ to 1.4 $\mathrm{mm}$, which results in an increase in force pulse from $4.3 \mathrm{pN}$ to $45 \mathrm{pN}$. The applied force leads to eight successive unfolding events observed as increase in step-size (defined as the difference between unfolded state and folded state of a domain). 

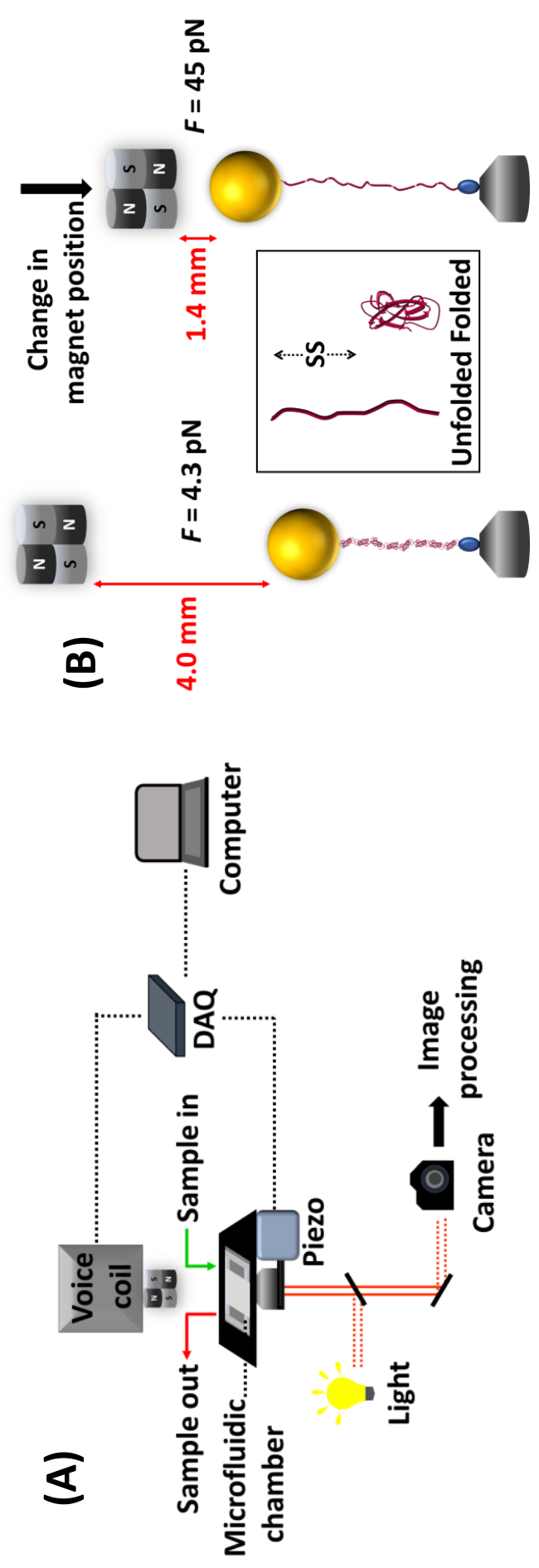

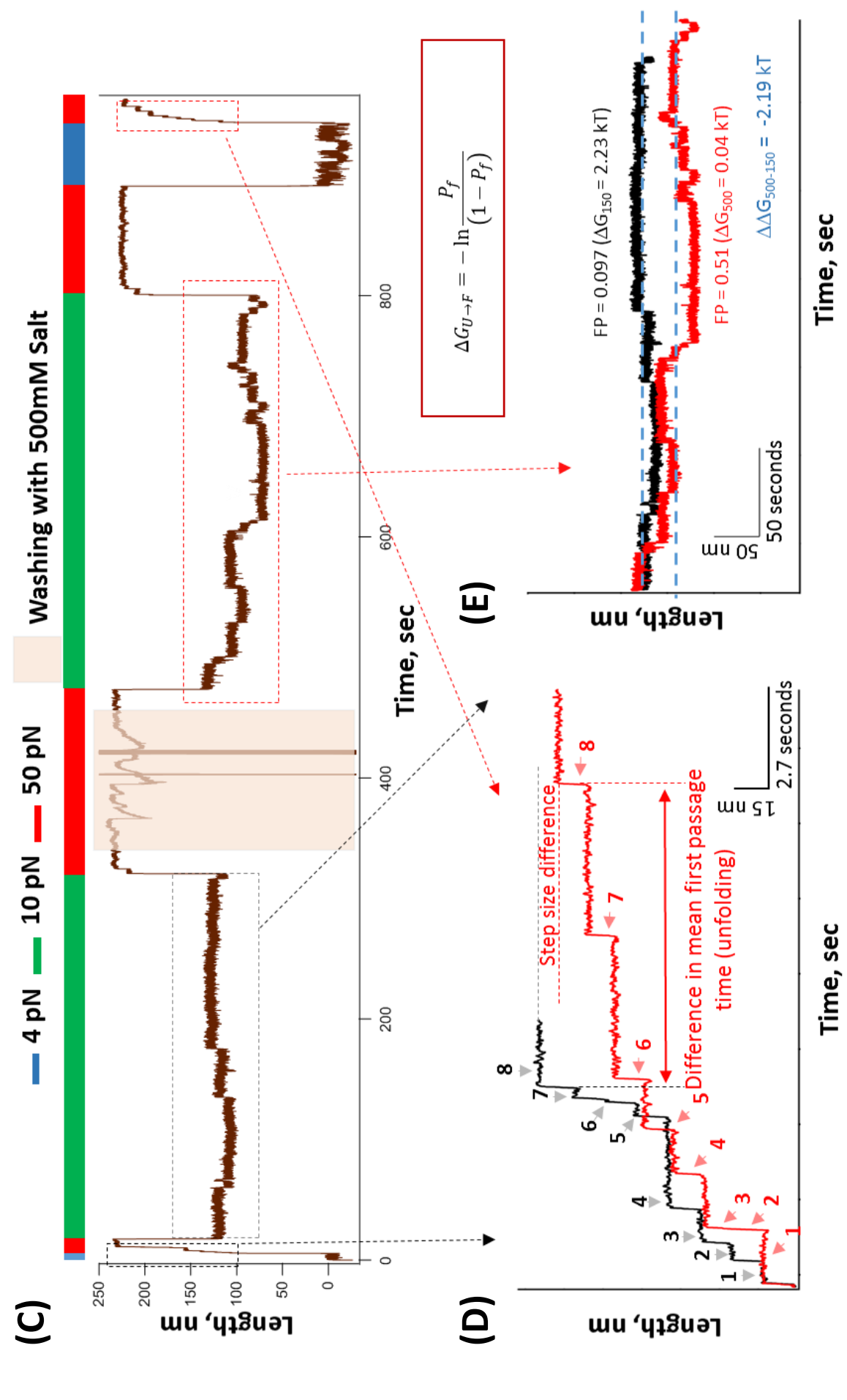


Figure 1: Real-time Multiplexed-Microfluidics-Magnetic Tweezers (RT-MMT): (A) RT-MMT instrumentation setup: The instrument includes a microfluidic chamber which ensures a continue flow of the buffer containing external stimuli and a covalently linked magnetic tweezers allowing us to change the solution multiple times during experiment while tracking a single protein molecule. The applied force on protein-bound paramagnetic bead is controlled by a pair of permanent magnets placed above the microfluidic chamber. (B) A schematic representation of RT-MMT experiment: The molecular construct comprising protein L octamer is flanked by an N-terminus HaloTag (blue sphere) for tethering to chloroalkane ligand on glass coverslip and C-terminus AviTag [HaloTag-(Protein L) 8-AviTag] for reacting to streptavidin coated paramagnetic bead (yellow sphere). The difference between unfolded and folded length is denoted as step-size (SS) (inset in Fig. 1B). (C) RT-MMT technology to monitor multiple events of protein folding in a single experiment: Firstly, the folded construct (at $4 p N$ ) is unfolded by a constant force of $50 \mathrm{pN}$ leading to eight successive unfolding steps as single molecule fingerprint. Then the force is allowed to quench at $10 \mathrm{pN}$ where the fully unfolded polypeptide shows immediate elastic recoiling and folding. Then the construct is unfolded by fingerprint pulse of $50 \mathrm{pN}$ and the flow cell is washed four times with $500 \mathrm{mM}$ salt. As previously, the force is quenched to $10 \mathrm{pN}$ for observing the equilibrium phase where any changes in folding-unfolding dynamics can be precisely traced and followed by similar high force pulse. Then the protein is completely refolded at $4 \mathrm{pN}$ and unfolded again at $50 \mathrm{pN}$ to check any changes in unfolding (D) Salt increases unfolding time and promotes conformational changes: The unfolding events at two different salt conditions are magnified and compared. At $150 \mathrm{mM}$, the octamer unfolds rapidly within $5.4 \mathrm{~s}$ but at $500 \mathrm{mM}$, it takes $13.45 \mathrm{~s}$. This delayed unfolding is a clear indication of higher mechanical stability of the folded protein provided by salt. Moreover, the observed difference in step size offers a hint of conformational changes. (E) Effect of salt at low force regime: The refolding trajectories at $150 \mathrm{mM}$ (black trace) and $500 \mathrm{mM}$ (red trace) are compared to investigate refolding time and folding probability. With $150 \mathrm{mM}$, the polyprotein shows folding-unfolding dynamics among $1^{\text {st }}, 2^{\text {nd }}$ folded state whereas with $500 \mathrm{mM}$, the dynamics is markedly accelerated and attain an equilibrium phase where it hops between $4^{\text {th }}, 5^{\text {th }}$ and $6^{\text {th }}$ folded states. Salt here works as a mechanical folder, allowing the protein to fold at high force

\section{A single real time tracking experiment of a single protein reports salt-dependent perturbation in kinetics, thermodynamics and polymer properties of protein $\mathrm{L}$ :}

Fig. 1C demonstrates how RT-MMT technology measures the difference in thermodynamic and polymer properties of a single protein in a single shot experiment, while introducing the salt during that experiment. In Fig. $1 \mathrm{C}$, at the first phase, the folding dynamics of protein $\mathrm{L}$ was recorded at $150 \mathrm{mM}$ salt and in the next phase, the microfluidic chamber was washed with $500 \mathrm{mM}$ salt, followed by measuring the folding dynamics of protein $\mathrm{L}$ in presence of $500 \mathrm{mM}$ salt. In this article we used ammonium sulfate $\left[\left(\mathrm{NH}_{4}\right)_{2} \mathrm{SO}_{4}\right]$ as a model salt.

In Fig. 1C, first protein $\mathrm{L}$ is unfolded at a high fingerprint force pulse of $50 \mathrm{pN}$ and subsequently quenched to equilibrium force of $10 \mathrm{pN}$ to monitor folding dynamics in presence of $150 \mathrm{mM}$ salt. This is followed by another high force pulse of $50 \mathrm{pN}$ and washed the flow cell four times with the $500 \mathrm{mM}(\mathrm{NH} 4)_{2} \mathrm{SO}_{4}$. The protein is then relaxed at $10 \mathrm{pN}$, unfolded at $50 \mathrm{pN}$, collapsed at 4 $\mathrm{pN}$ and again unfolded at $50 \mathrm{pN}$. Notably, the covalent bond between HaloTag enzyme and chloroalkane ligand helps the protein to remain tethered to the glass surface while washing the flow cell. Two unfolding trajectories (from $4 \mathrm{pN}$ to $50 \mathrm{pN}$ ) at $150 \mathrm{mM}$ and $500 \mathrm{mM}$ salt, are magnified and compared in Fig. 1D. In $150 \mathrm{mM}$ salt, the protein L construct unfolds rapidly within $5.4 \mathrm{~s}$ (Fig. 1D, black trace), whereas with $500 \mathrm{mM}$, the unfolding becomes retarded to $13.45 \mathrm{~s}$ (Fig. 
1D, red trace). This result clearly demonstrates the increase in unfolding time with salt concentration due to more stable folded state. Furthermore, the same data also showed a prominent decrease in step size, which argues in favor of salt dependent conformational change in protein.

Similarly, from Fig. 1C, equilibrium phases (at $10 \mathrm{pN}$ ) are zoomed in to show the comparison between folding probability $(F P)$ and refolding kinetics in different salt concentration (Fig. 1E). After using the extended pulse to unfold, the polyprotein is allowed to fold by quenching the force to $10 \mathrm{pN}$ at both salt concentrations. Upon addition of $500 \mathrm{mM}$ salt to flow cell, folding probability is altered in favor of folded state. During the first equilibrium trajectory with $150 \mathrm{mM}$ salt, the polyprotein hops between fully unfolded, $1^{\text {st }}$ and $2^{\text {nd }}$ folded states to give a calculated value of FP $=0.097$ (Fig. 1E, black trace), whereas after introducing $500 \mathrm{mM}$ salt, the polyprotein hops between $4^{\text {th }}, 5^{\text {th }}$ and $6^{\text {th }}$ folded state by accelerating its $F P$ to 0.51 (Fig. 1E, red trace). We have examined the folding probability by dwell-time analysis (supplementary table 1 ). In equilibrium phase, each folded state is denoted as $I$ ( $I$ is the number of folded states) and their residence time along the phase is calculated as $t=T_{I} / T_{T}$, where $T_{I}$ is the time spent on I state and, $T_{T}=\sum_{I} T_{I}$, total observation time at equilibrium. The folding probability $(F P)$ is measured as normalized averaged state,

$F P=\sum_{I} I * t / N$

Where $N=8$ is equivalent to total numbers of domains. Detailed calculations of $F P$ have been discussed in supplementary table. 1 :

$$
\Delta G_{U \rightarrow F}=-\ln \frac{F P}{(1-F P)}
$$

We obtained the equilibrium free energy difference for folding $\left(\Delta \Delta \mathrm{G}_{\mathrm{U} \rightarrow \mathrm{F}}\right)$ from the same experiment by analyzing the equilibrium folding under constant force of $10 \mathrm{pN}$ with the help of eq. 1 and eq. 2 . We estimated the free energy in presence of $150 \mathrm{mM}$ salt $\left(\Delta \mathrm{G}_{150}\right)$ is $2.23 \mathrm{kT}$ and that of $500 \mathrm{mM}\left(\Delta \mathrm{G}_{500}\right)$ salt is $0.04 \mathrm{kT}$. Taken together, the $\Delta \Delta \mathrm{G}_{\mathrm{U} \rightarrow \mathrm{F}}\left(\Delta \mathrm{G}_{500}-\Delta \mathrm{G}_{150}\right)$ is around $-2.19 \mathrm{kT}$ which shows the folding process is more favored in presence of $500 \mathrm{mM}$ salt. Moreover, from the Fig. 1E we can compare the refolding kinetics. In presence of $150 \mathrm{mM}$, two domains fold in 140 seconds but in $500 \mathrm{mM}$ salt, six domains fold within the same observed time scale.

\section{Comparison of single experiment data with many single molecule experiments:}

In Fig. 2, the information obtained from the single experiment (Fig. 1C) is correlated with the results of many single molecule experiments at a constant salt concentration. In this figure many folding dynamics events of protein $\mathrm{L}$ were monitored at a constant salt concentration and their average results are compared with the data obtained from the single experiment (Fig. 1C).

Fig. 2A shows a standard experimental trajectory of an octamer construct of protein L, where the construct is unfolded by an unfolding pulse of $45 \mathrm{pN}$ force and folded by a subsequent refolding pulse of $9 \mathrm{pN}$. After applying the refolding pulse, the domains undergo an equilibrium stage, where 
the domains exhibit folding/unfolding dynamics as ascending/descending steps. The unfolding trajectory is magnified in the inset of Fig. 2A, where the first passage time (FPT) of unfolding is described - the minimum time taken to complete unfolding from fully folded state (time between the arrow, in Fig. 2A inset). Similarly, the FPT of refolding is calculated as time for a complete refolding from a complete unfolded state. Mean first passage time (MFPT) is determined by averaging the FPT values over several trajectories, offering a model-free metric that describes the unfolding and refolding kinetics of protein under force. Fig. 2B shows the variation of MFPT of unfolding as a function of force and salt concentration. At a particular salt concentration, the MFPT of unfolding decreases with increasing mechanical force - showing the inverse relation of protein folding with force. However, salt changes the energy landscape and unfolding time increases with salt concentration such as, at $50 \mathrm{pN}$, complete unfolding takes $5.5 \pm 2.1 \mathrm{~s}$ in presence of $150 \mathrm{mM}$ salt whereas it increases to $12.48 \pm 1.6$ with $500 \mathrm{mM}$ salt. Importantly, our single molecule data is strongly correlated with the unfolding-MFPT plot such as, in presence of $150 \mathrm{mM}$ salt, complete unfolding of eight domains at $50 \mathrm{pN}$ takes only $5.4 \mathrm{~s}$ (Fig. 2B, pink triangle) but at $500 \mathrm{mM}$, the time shifts to $13.45 \mathrm{~s}$, (Fig. 2B, black triangle). In contrast to MFPT of unfolding, MFPT of refolding decreases with increase in salt concentration. For example, at $9 \mathrm{pN}$ force with $150 \mathrm{mM}$ salt, the polyprotein completely refolds at $275 \pm 10 \mathrm{~s}$ (Fig. 2C, black trace) whereas in presence of $650 \mathrm{mM}$ salt, complete refolding takes just $43 \pm 14 \mathrm{~s}$ (Fig. 2C, red trace). Thus, salt delays the unfolding kinetics and accelerates the refolding kinetics in concentration dependent manner. 


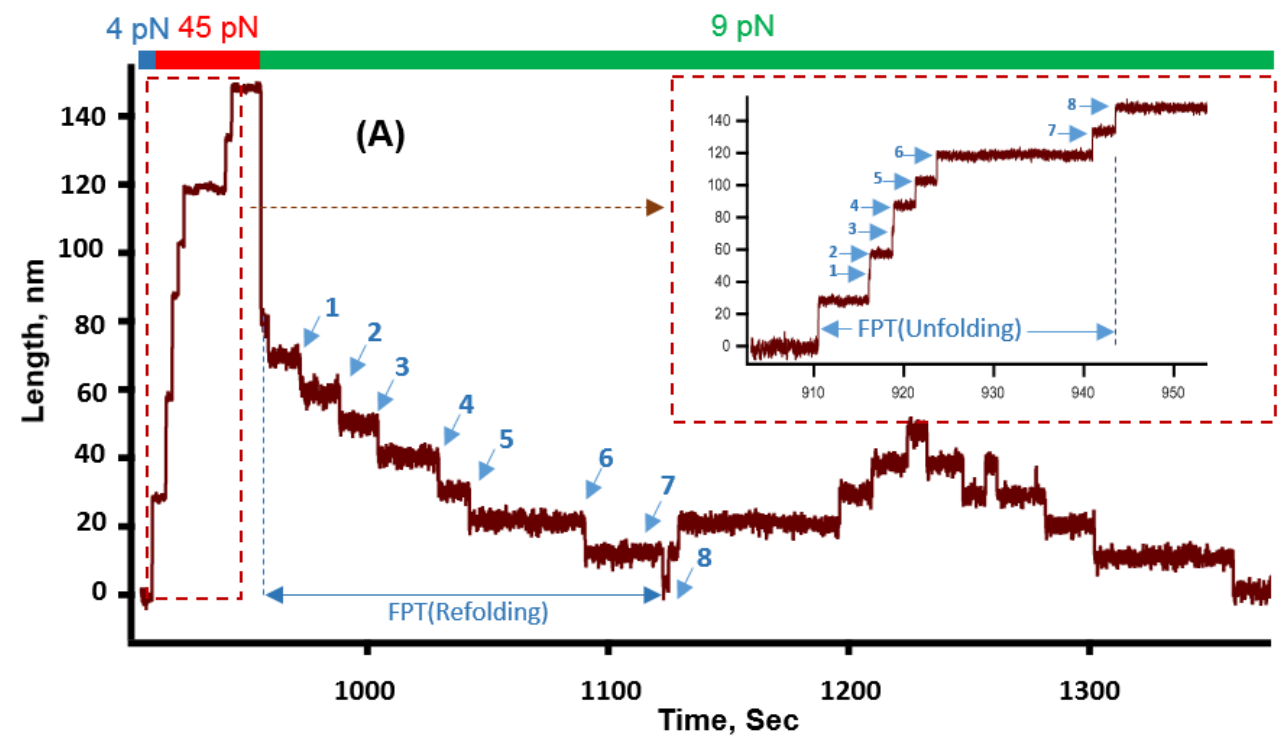

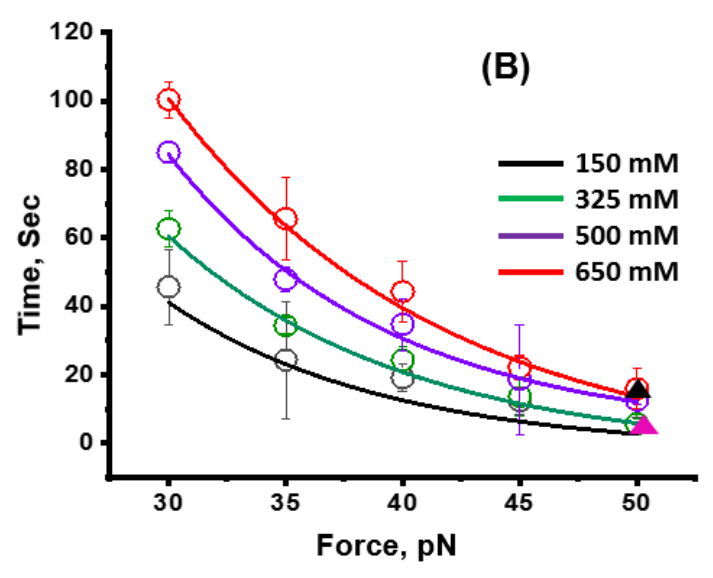

(D)

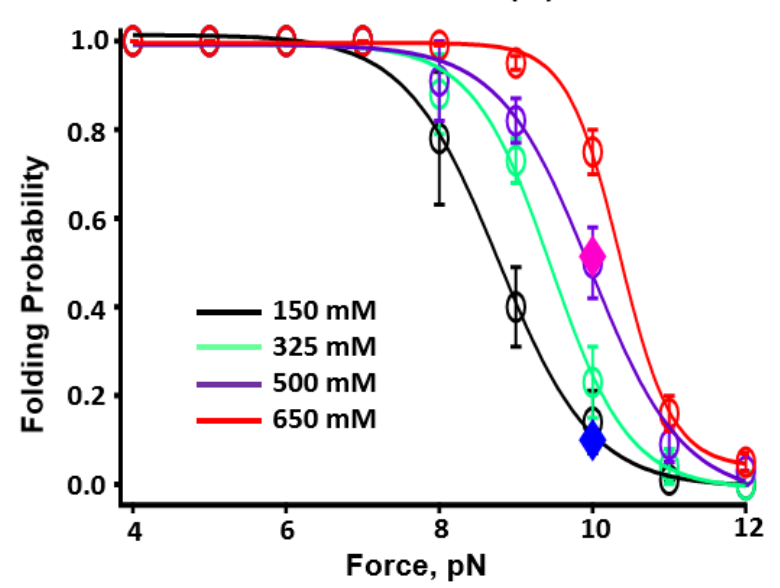

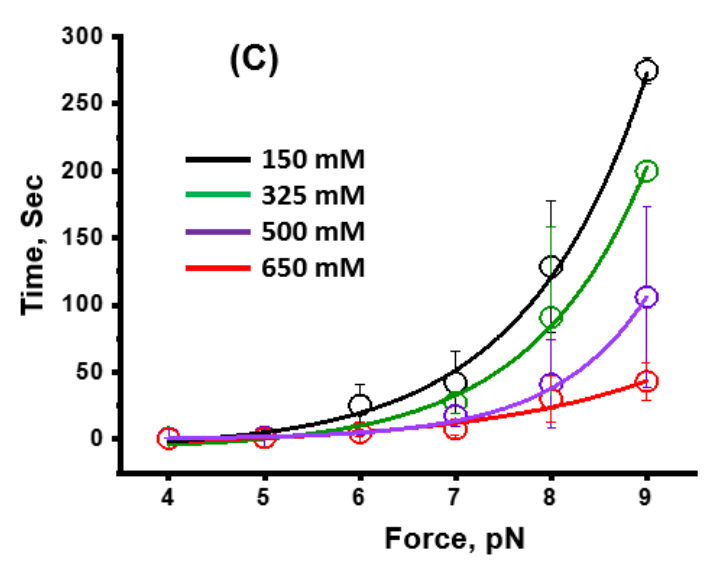

(E)

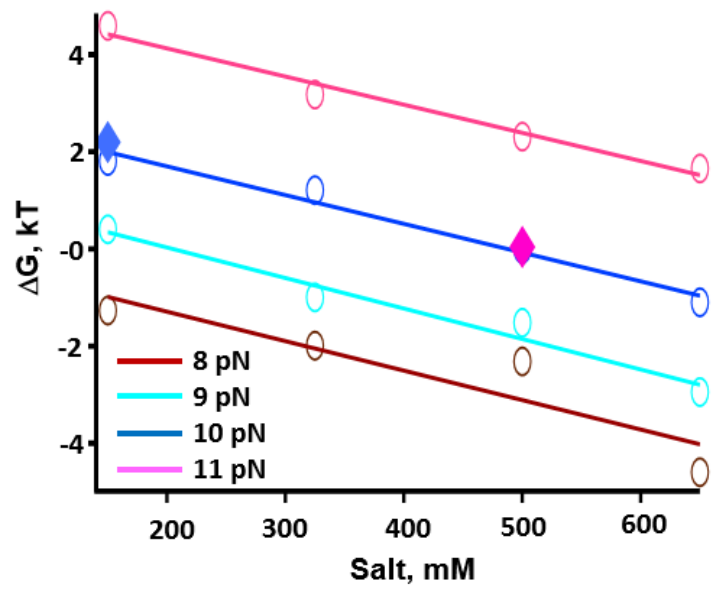


Figure 2: Comparison of single experiment with many single molecule experiments: (A) Standard trajectory obtained from $\mathbf{R T}-\mathbf{M M T}$ experiment: The protein L octamer is first unfolded at a fingerprint pulse of $45 \mathrm{pN}$ leading to complete unfolding of eight domains. After unfolding, the force is quenched to 9 $p N$ for observing the folding-unfolding dynamics at equilibrium phase. The unfolding trajectory is magnified to inset, where the first passage time of unfolding (FPT) is described as the time taken to complete unfolding from fully folded state. Similarly, FPT of refolding denotes the total time for complete refolding from fully extended state. (B) Variation of mean-FPT for unfolding: The MFPT of unfolding is plotted as a function of force with different salt conditions. The unfolding time decreases steadily with increasing force, however, salt, acting against to force, increases the time for complete unfolding. Notably, the data at $50 \mathrm{pN}$, acquired from single molecule analysis, shows complete unfolding with $150 \mathrm{mM}$ takes $5.4 \mathrm{~s}$ (pink triangle), whereas it increases to $13.44 \mathrm{~s}$ with $500 \mathrm{mM}$ (black triangle) which are consistent to the plot of unfolding-MFPT. (C) Variation of MFPT for refolding: Salt reduces the total refolding time. For example, at $9 \mathrm{pN}$, in presence of $150 \mathrm{mM}$, the MFPT of refolding is $275 \pm 10$ s (black trace) while with $650 \mathrm{mM}$ the time decreases to $43 \pm 14 \mathrm{~s}$ (red trace). Error bars are s.e.m. (D) Folding probability as a function of salt concentration: The folding probability as a function of force is plotted for four different salt concentrations $(150,325,500,650 \mathrm{mM})$. The effects of salt on folding probability increases considerably over a range 8 $11 \mathrm{pN}$ and attains maximal at $10 \mathrm{pN}$ force. Our single experiment showed that at $10 \mathrm{pN}$, the folding probability has been increased from 0.097 (in $150 \mathrm{mM}$ salt, blue diamond) to 0.51 (in $500 \mathrm{mM}$ salt, pink diamond), showing a strong concentration dependent role of salt on protein folding. Error bars represent s.e.m. (E) Effect on $\Delta \boldsymbol{G}_{\text {folding }}$ with increasing salt concentration: $\Delta G_{\text {folding }}(k T)$ is plotted as a function of salt concentration at different forces ranging from 8 to $11 \mathrm{pN}$. With increasing salt concentration at a particular force, the $\Delta G$ decreases. From our single experiment data of folding probability, we calculated the $\Delta G$ at $10 \mathrm{pN}$ force as $2.23 \mathrm{kT}(150 \mathrm{mM}$, blue diamond) and $0.04 \mathrm{kT}$ (500 $\mathrm{mM}$, pink diamond), which are precisely coinciding with our $\Delta G$ plot.

Similar to MFPT for unfolding and refolding, salt concentration greatly affects the thermodynamic energy landscape of protein folding, as observed in Fig. 1E. To thoroughly investigate that, we plot the folding probability as a function of force at different salt conditions. We observed that folding probability decreases with increasing force - showing again the inverse relation of folding and force. However, introducing salt, opposing to force, helps in protein folding and thus, escalates the folding probability. At 4-7 $\mathrm{pN}$ force regime, all domains fold rapidly and completely, irrespective to the salt concentration and thus, $\mathrm{FP}=1$. At $12 \mathrm{pN}$, the FP decreased to zero, except 500 and $650 \mathrm{mM}$ where FP are $0.03 \pm 0.02$ and $0.05 \pm 0.02$, respectively. However, at an intermediate force range of $8-11 \mathrm{pN}$, the folding probability is drastically raised with increasing salt (Fig. 2D). The half-point force (defined as force where FP $=0.5$ ) shifted rightward from $\sim 8.8$ to $\sim 10.4 \mathrm{pN}$ at $150 \mathrm{mM}$ to $650 \mathrm{mM}$ range, showing a higher intrinsic ability of protein to remain folded against the force in presence of salt. Fig. $2 \mathrm{E}$ shows a comparative data of free energy difference $(\Delta \mathrm{G})$ within a span of 8-11 pN force, where both folding-unfolding transitions occur within experimental time-scale and $\Delta \mathrm{G}_{\mathrm{U} \rightarrow \mathrm{F}}$ was calculated from eq. 2. Our result shows salt favors folding and thus, $\Delta \Delta \mathrm{G}_{\mathrm{U} \rightarrow \mathrm{F}}$ decreases with gradual increment in salt concentration and the result is consistent for all the force range from $8-11 \mathrm{pN}$. Indeed, these results are in agreement with our single experiment data shown in blue $(150 \mathrm{mM})$ and pink $(650 \mathrm{mM})$ diamond in Fig $2 \mathrm{D}$ and 2E. 


\section{Conformational compaction of protein described by 'electrolyte FJC model':}

Although salt effects on protein folding is thoroughly characterized, how electrolytes influence conformational flexibility under force is not known. To systematically investigate that, we monitored the salt dependent conformational changes of a single protein in a wide force range. With increasing salt concentration at a particular force, we observed a gradual decrease in step sizes (Fig. 3). We determined the Kuhn length (Supplementary Table 2) and Kuhn segment (Table 1) for protein $L$ at different salt concentration by plotting the step size data to freely jointed chain (FJC) model (eq. 3) against different forces at constant salt concentration ${ }^{15,16}$. The protein is considered as linear single stranded polymer having maximum extended length $\left(L_{c}\right.$, contour length) with $N$ numbers of uncorrelated Kuhn segments of length $L_{k}$ (Kuhn length) where, $L_{C}=$ $N L_{k}$ and each of those segments can take any 3D orientation. Considering $L_{c}$ of $16.3 \mathrm{~nm}$ for protein $\mathrm{L}^{17}, N$ is calculated and shown in Table 1 .

$$
S S=L_{c} \times\left[\left\{\operatorname{coth}\left(\frac{F L_{k}}{k T}\right)\right\}-\left\{\frac{k T}{F L_{k}}\right\}\right]
$$

Where, $S S$ is step size or extension; $F$ is force $(\mathrm{pN}) ; k T$ is thermal energy and has a value of 4.11 pN. nm and $N$ is the number of uncorrelated Kuhn segments of length $L_{k}$ where, $L_{C}=N L_{k}$ and each of those segments can take any 3D orientation.

\begin{tabular}{|c|c|}
\hline Salt Concentration $(\mathbf{m M})$ & Kuhn segments $(\mathbf{N})$ \\
\hline 150 & $13.81 \pm 0.61$ \\
\hline 325 & $15.52 \pm 0.15$ \\
\hline 500 & $19.92 \pm 0.28$ \\
\hline 650 & $28.10 \pm 0.50$ \\
\hline
\end{tabular}

Table 1: Variation in Kuhn segments with increasing salt concentration

We observed a ln-dependent Kuhn length change with salt concentration ${ }^{11,18}$ and explained as $L_{k}=a \ln \frac{c_{0}}{c}(4)$, which modifies the FJC model (mod-FJC) to determine the SS value as a function of salt concentration, $c$,

$$
S S=L_{c} \times\left[\left\{\operatorname{coth}\left(\frac{F\left(a \ln \frac{c_{0}}{c}\right)}{k T}\right)\right\}-\left\{\frac{k T}{F\left(a \ln \frac{c_{0}}{c}\right)}\right\}\right]
$$

Where $\mathrm{a}$ is the Kuhn length of protein $\mathrm{L}$ and the relation works in the range of $150-650 \mathrm{mM}$ ammonium sulfate salt concentration. 

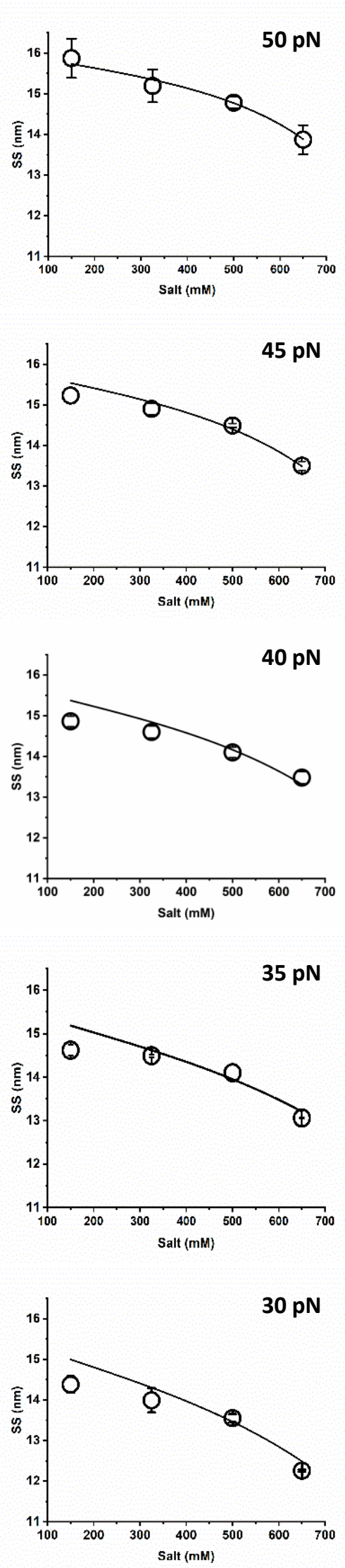

Figure 3: Salt mediated conformational changes of protein L: At particular force, the step sizes decreased consistently with increasing salt concentration, which can be fitted with the modified FJC mode (eq. 5). This decrease in step size with salt concentration is generic and force independent.

\section{Conformational change of protein $L$ is not force dependent:}

To exclude any artefactual effect, coming from the force or attaching principle, in the measurement of conformational change, we studied the diffusion time of the freely diffusing protein $\mathrm{L}$ (same construct) by fluorescence correlation spectroscopy (FCS) and determine its diffusion time $\left(\tau_{\mathrm{D}}\right)$ using eq. $6^{19,20}$. The $\tau_{\mathrm{D}}$ values are used to denote the time, taken by a molecule, while freely diffusing in and out of the observation volume (Fig. 4A). Diffusion time of the freely diffusing protein is proportional to its hydrodynamic radii $\left(r_{H}\right)$ (eq. 7-9) and thus decrease in diffusion time describes the compactness of the biomolecules with increase in the salt concentration (Fig. 4B).

$$
G(\tau)=1+\frac{1}{N_{P}}\left(\frac{1}{1+\frac{\tau}{\tau_{D}}}\right)\left(\frac{1}{1+S^{2} \frac{\tau}{\tau_{D}}}\right)^{0.5}
$$

Where $G(\tau)$ is the correlation function, $N_{P}$ is the particle number in the observation volume and $S$ is the structure parameter that is denoted as the ratio of depth to diameter of Gaussian observation volume. Diffusion coefficient $(D)$ can be calculated from $\tau_{D}$ from eq. 7:

$\tau_{D}=\frac{\omega^{2}}{4 D}$

Where $\omega$ is the beam radius of observation volume and thus, $\tau_{D}$ and $D$ satisfy an inverse relationship, $\tau_{D} \propto 1 / D$. Subsequently, hydrodynamic radius $\left(r_{H}\right)$ of the TMR-labeled protein molecule is calculated by Stokes-Einstein equation as eq. 8 :

$D=\frac{k T}{6 \pi \eta r_{H}}$

Where kT is thermal energy, $\eta$ is viscosity of solution and it ultimately makes: $D \propto 1 / r_{H}$ thus, the relation between $\tau_{D}$ and $r_{H}$ can be expressed as eq. 9 :

$\tau_{D} \propto r_{H}$ 

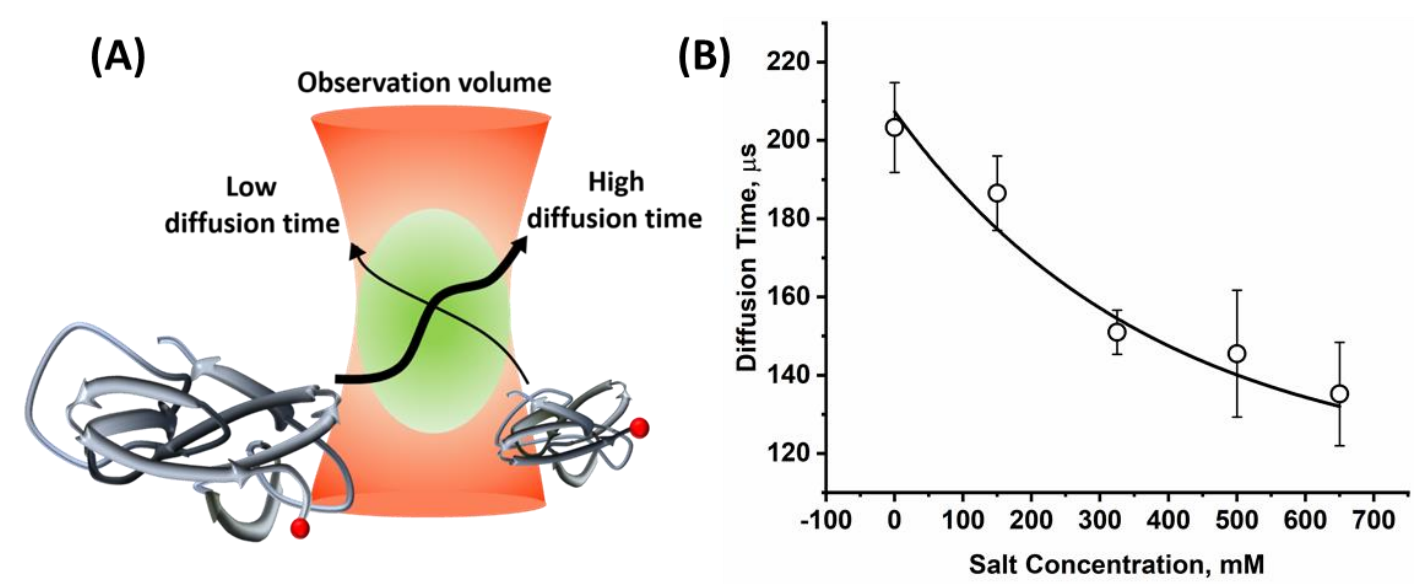

Figure 4: FCS measurements of TMR-labeled Protein L: (A) Schematic representation of FCS measurement: In FCS experiment, diffusion time is quantified as the time, a molecule takes to diffuse in and out of the observation volume. If the fluorophore labeled-molecule is small, it diffuse faster through observation volume and thus, $\tau_{D}$ decreases. (B) Salt dependent variation in diffusion time $\left(\tau_{D}\right)$ of protein L: With increase in salt concentration the diffusion time of protein L decreases, such as, in absence of salt, the $\tau_{D}$ is $203.3 \pm 11.5 \mu$ s but it decreases to $135.2 \pm 13.2 \mu$ s in presence of $650 \mathrm{mM}$ salt.

\section{Discussion}

The single molecule analysis, performed by RT-MMT technology, reveals an integrated approach of studying protein folding by combining kinetics and thermodynamics to polymer physics from a single experiment (Fig. 5). Here we monitored both mechanical unfolding and refolding at varying salt concentrations in a wide range of mechanical force (Fig 5A). Our result shows, salt escalates the conformational flexibility by increasing the number of Kuhn segments, promoting the higher degrees of native contacts to favor the folding (Fig. 5B and 5C), which also reflects in the decrease of MFPT $_{\text {Refolding }}$ and increase of MFPT $_{\text {Unfolding. Additionally, from the folding probability and }}$ MFPT calculations, we found how salt changes the energy landscape by stabilizing the folded state and destabilizing the unfolded state (Fig. 5D). Ammonium sulfate solution, as a poor solvent, collapses the polypeptide in a concentration dependent manner due to an enhanced monomermonomer interaction and a diminished monomer-solvent interaction. This can also be explained from smaller excluded volume which further reduces radius of gyration $\left(R_{g}\right)^{21}$. Our work is coinciding with simulation study where it has been shown that poor solvents redesign the Ramachandran plot by redistributing polypeptide dihedral angles $\varphi$ and $\psi$ to largely attribute in the fold topology during the early collapse in the folding pathway. Indeed, the 'bridge region' between the $\beta$-basin and $\alpha$ region in Ramachandran plot is not allowed in good solvents while in poor solvent the bridge region is favored and thus, induces the folding during molecular compaction of denatured state ensemble ${ }^{22-24}$. The resulting molecular compaction facilitates the protein folding by decreasing both their conformational entropy and conformational spaces, screened during folding. Though the nature of salt mediated compaction is still elusive with plausible contribution 
from either unfolded state compaction or that of folded state or even from both the states, chain compaction into a very dense state diminishes the diffusive chain fluctuations by inducing internal friction that increases with elevated compaction of unfolded proteins ${ }^{25,26-30}$. The unfolded state of protein $\mathrm{L}$ molecule undergoes rapid compaction in folding condition with $\sim 10 \%$ larger than its folded state which apparently suggests the compaction of unfolded state ${ }^{31}$. Additionally, in poor solvent, the protein behaves like heteropolymers, resulting in an initial collapse followed by secondary structure formation mainly arisen due to specific contacts which in turn greatly speed up the folding. Similarly, we observed ionic strength favors protein folding phenomena by concomitantly increasing the native contacts during structure formation and facilitating the thermodynamics of folding (Fig. 5C) which strongly reconciles the compaction driven protein folding.

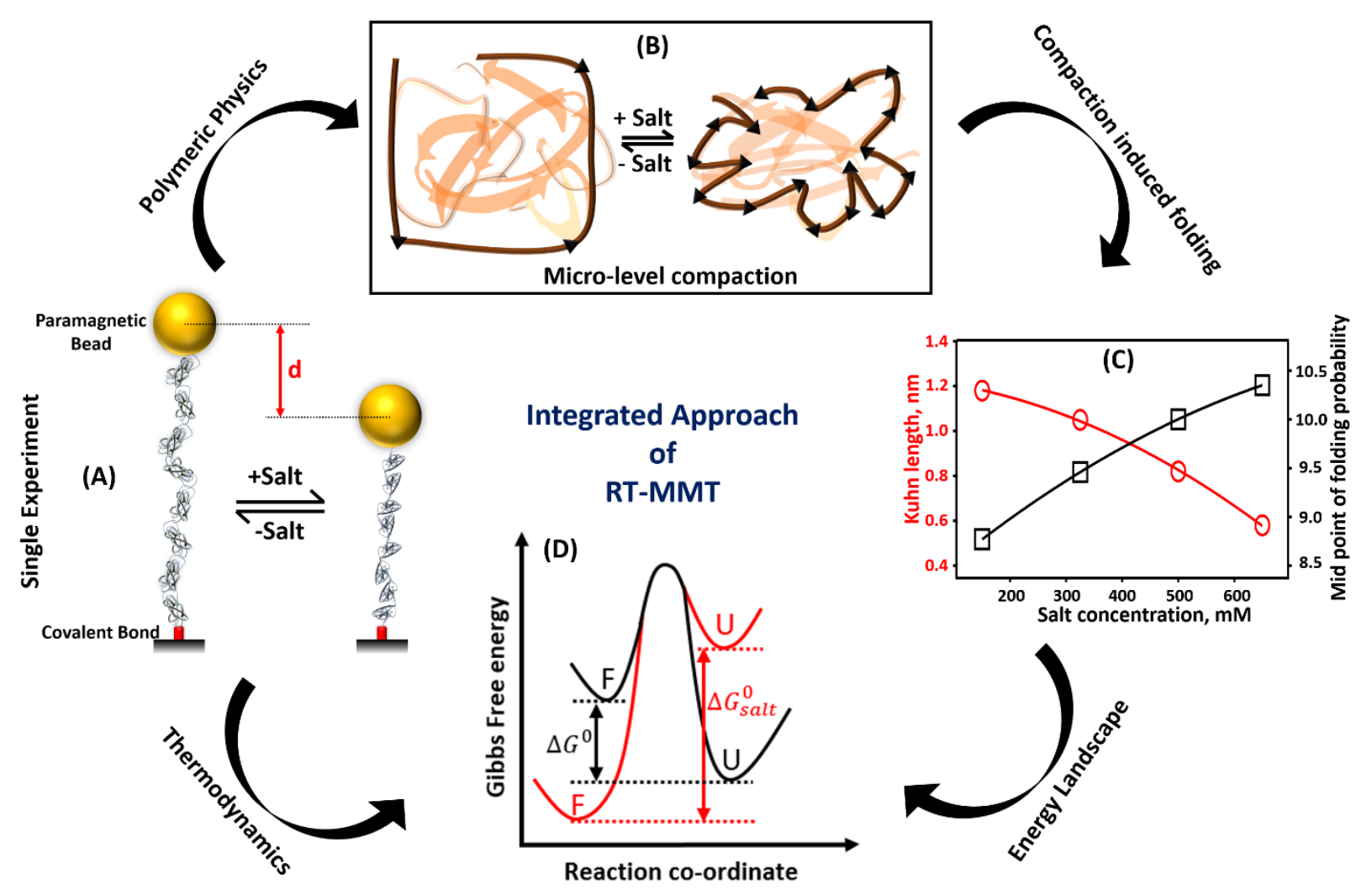

Figure 5: RT-MMT has a multifaceted application to integrate polymer physics, kinetics and thermodynamics under the single experiment: (A) Salt induces the conformational changes in protein domains that observed from the decrease in step-size 'd'. (B) Salt induced change in polymer property: Addition of salt causes compaction of polypeptide chain. This compaction can be analyzed by illustrating the micro-level compaction, where salt increases the number of Kuhn segment in protein polymer to promote flexibility. (C) Molecular compaction facilitates protein folding: Kuhn length (red circle, supplementary table. 2) and mid-point of folding probability (black square, Figure. 2D) are plotted as a function of salt concentration. Salt decreases the Kuhn length of the protein to induce the mechanical flexibility while simultaneously increases the mid-point of folding probability promoting a higher intrinsic 
ability to remain in the folded state. Thus, higher order of native contacts and successive molecular compaction, due to increased mechanical flexibility, facilitates the stability of the folded state. (D) Reshaping of the energy landscape of protein $L$ : salt addition redesigns the free energy landscape by stabilizing folded state and destabilizing unfolded state and thus, increases the difference in $\Delta G$ between folded and unfolded state.

In cells, ion homeostasis is pivotal to maintain cellular functions by constantly changing the ionic environment that affect the elasticity of different biological polymers such as, DNA, RNA or proteins. Although polymer properties of nucleic acids, being flexible polyelectrolytes, have been tremendously studied ${ }^{11,13,32-40}$, no such behavior of proteins has been documented yet, especially how the ion driven conformational flexibility of proteins changes under force. To obtain deeper insights into this, we characterize the protein $\mathrm{L}$ as a polymer where their step-sizes are described by the simple FJC model. Since, the $L_{c}$ and $L_{k}$ are known to be fixed for a protein polymer; the existing FJC model only describes the step-sizes as a function of force ${ }^{15,16}$. We have modified the FJC model to analyze the conformational compaction of protein polymer in varying ionic environment. Indeed, the mechanosensing polyprotein like microtubules and actin have relatively higher persistence length $\left(L_{p}\right)$ and thus show higher bending rigidity that allows them to form crosslinking structures ${ }^{41,42}$ in cell cortex and protrusion. Moreover, due to extended topology they are constantly exposed to the varying ionic environments that change unfolding pattern of their domains during interaction with other proteins that further can decode unprecedented physical viewpoints of cell division and structure maintenance. Interestingly, post-translational modifications in polypeptides change their resultant charge distribution that alters their elasticity and end-to-end distance. ${ }^{43}$ In physical perspective, the electrostatic interaction among charges in a polypeptide is regulated by ionic strength which effectively alters their electrostatic contribution to $L_{k}$ or $L_{p}$ by changing Debye screening length $\left(\lambda_{D}\right)$. Interestingly, the cellular compartments involved in secretory pathway have a well-defined $\mathrm{pH}$ continuum from the neutral $\mathrm{pH}$ to acidic $\mathrm{pH}$ that could affects the folding and stability of secreting protein as well as resident proteins. Illustrating their altered polymer properties in dynamic ionic environment could reveal new horizon for studying protein functions.

Comparative analysis of molecular properties, before and after interaction, is appropriate if they could be performed by a single technique - as every methodology requires different chemical or physical manipulations, which introduce associated artifacts. The comparison could be even more precise if carried out on the same molecule. Here we compare five molecular properties by a single technology on a single protein molecule. This integrated approach quantifies both the elastic properties and unfolding-refolding dynamics simultaneously from a single experiment. By correlating both these molecular properties from the one single experiment, we directly observe the conformational compactness of a protein modifies its energy landscape. In future, this methodology could also have translational application to use as a high throughput single molecule microfluidic assay for drug screening (Supplementary Fig. 1). Finally, development of RT-MMT 
with other super-resolution technology will guide us to illustrate diverse cellular processes by imaging under force, bridging the concepts of physics and biology.

\section{Acknowledgement:}

We thank Ashoka University for support and funding. S.H. thanks DBT Ramalingaswami Fellowship and DST SERB Core Research Grant for funding. We thank Prof. Julio Fernandez (Columbia University) for helping us with the magnetic tweezers set up and Dr. Krishnananda Chattopadhyay (IICB) for the FCS measurements. We thanks Dr. Rafael Tapia-Rojo (Columbia University), Dr. Edward C Eckels (Columbia University), Prof. Somendra M. Bhattacharjee (Ashoka University) and Prof. Gautam Menon (Ashoka University) for the critical analysis on the manuscript.

\section{Conflict of Interest:}

The authors declare no conflict of interest.

\section{References:}

1. Rai, A. K., Rai, A., Ramaiya, A. J., Jha, R. \& Mallik, R. Molecular Adaptations Allow Dynein to Generate Large Collective Forces inside Cells. Cell 152, 172-182 (2013).

2. Imanishi, M., Endres, N. F., Gennerich, A. \& Vale, R. D. Autoinhibition regulates the motility of the C. elegans intraflagellar transport motor OSM-3. J. Cell Biol. 174, 931-937 (2006).

3. Kiefhaber, T. Kinetic traps in lysozyme folding. Proc. Natl. Acad. Sci. U. S. A. 92, 9029-9033 (1995).

4. Velazquez-Campoy, A. \& Freire, E. Isothermal titration calorimetry to determine association constants for high-affinity ligands. Nat. Protoc. 1, 186-191 (2006).

5. Tapia-Rojo, R., Eckels, E. C. \& Fernández, J. M. Ephemeral states in protein folding under force captured with a magnetic tweezers design. Proc. Natl. Acad. Sci. 116, 7873-7878 (2019).

6. Suren, T. et al. Single-molecule force spectroscopy reveals folding steps associated with hormone binding and activation of the glucocorticoid receptor. Proc. Natl. Acad. Sci. 115, 11688-11693 (2018).

7. Schindler, T., Herrler, M., Marahiel, M. A. \& Schmid, F. X. Extremely rapid protein folding in the absence of intermediates. Nat. Struct. Biol. 2, 663-673 (1995).

8. Saio, T., Guan, X., Rossi, P., Economou, A. \& Kalodimos, C. G. Structural basis for protein antiaggregation activity of the trigger factor chaperone. Science 344, 1250494 (2014).

9. Peng, Q. \& Li, H. Atomic force microscopy reveals parallel mechanical unfolding pathways of T4 lysozyme: Evidence for a kinetic partitioning mechanism. Proc. Natl. Acad. Sci. 105, 18851890 (2008).

10. Greenfield, N. J. Using circular dichroism collected as a function of temperature to determine the thermodynamics of protein unfolding and binding interactions. Nat. Protoc. 1, 2527-2535 (2006).

11. Manning, G. S. The Persistence Length of DNA Is Reached from the Persistence Length of Its Null Isomer through an Internal Electrostatic Stretching Force. Biophys. J. 91, 3607-3616 (2006). 
12. Chen, H. et al. Ionic strength-dependent persistence lengths of single-stranded RNA and DNA. Proc. Natl. Acad. Sci. 109, 799-804 (2012).

13. Baumann, C. G., Smith, S. B., Bloomfield, V. A. \& Bustamante, C. Ionic effects on the elasticity of single DNA molecules. Proc. Natl. Acad. Sci. 94, 6185-6190 (1997).

14. Haldar, S. \& Chattopadhyay, K. Interconnection of Salt-induced Hydrophobic Compaction and Secondary Structure Formation Depends on Solution Conditions REVISITING EARLY EVENTS OF PROTEIN FOLDING AT SINGLE MOLECULE RESOLUTION. J. Biol. Chem. 287, 11546-11555 (2012).

15. Flory, P. J. Principles of Polymer Chemistry. (Cornell University Press, 1953).

16. Eckels, E. C., Haldar, S., Tapia-Rojo, R., Rivas-Pardo, J. A. \& Fernández, J. M. The Mechanical Power of Titin Folding. Cell Rep. 27, 1836-1847.e4 (2019).

17. Haldar, S., Tapia-Rojo, R., Eckels, E. C., Valle-Orero, J. \& Fernandez, J. M. Trigger factor chaperone acts as a mechanical foldase. Nat. Commun. 8, 668 (2017).

18. Manning, G. S. A procedure for extracting persistence lengths from light-scattering data on intermediate molecular weight DNA. Biopolymers 20, 1751-1755 (1981).

19. Ghosh, R., Sharma, S. \& Chattopadhyay, K. Effect of Arginine on Protein Aggregation Studied by Fluorescence Correlation Spectroscopy and Other Biophysical Methods. Biochemistry 48, 1135-1143 (2009).

20. Haldar, S., Mitra, S. \& Chattopadhyay, K. Role of Protein Stabilizers on the Conformation of the Unfolded State of Cytochrome c and Its Early Folding Kinetics INVESTIGATION AT SINGLE MOLECULAR RESOLUTION. J. Biol. Chem. 285, 25314-25323 (2010).

21. Wilkins, D. K. et al. Hydrodynamic radii of native and denatured proteins measured by pulse field gradient NMR techniques. Biochemistry 38, 16424-16431 (1999).

22. Porter, L. L. \& Rose, G. D. Redrawing the Ramachandran plot after inclusion of hydrogenbonding constraints. Proc. Natl. Acad. Sci. 108, 109-113 (2011).

23. Bowler, B. E. Residual structure in unfolded proteins. Curr. Opin. Struct. Biol. 22, 4-13 (2012).

24. Gong, H., Porter, L. L. \& Rose, G. D. Counting peptide-water hydrogen bonds in unfolded proteins. Protein Sci. Publ. Protein Soc. 20, 417-427 (2011).

25. Am, G., Vi, A. \& Ei, S. Is Burst Hydrophobic Collapse Necessary for Protein Folding? Biochemistry vol. 34 https://pubmed.ncbi.nlm.nih.gov/7893719/?dopt=Abstract (1995).

26. Hofmann, H. et al. Polymer scaling laws of unfolded and intrinsically disordered proteins quantified with single-molecule spectroscopy. Proc. Natl. Acad. Sci. 109, 16155-16160 (2012).

27. Möglich, A., Joder, K. \& Kiefhaber, T. End-to-end distance distributions and intrachain diffusion constants in unfolded polypeptide chains indicate intramolecular hydrogen bond formation. Proc. Natl. Acad. Sci. 103, 12394-12399 (2006).

28. Nettels, D., Gopich, I. V., Hoffmann, A. \& Schuler, B. Ultrafast dynamics of protein collapse from single-molecule photon statistics. Proc. Natl. Acad. Sci. 104, 2655-2660 (2007).

29. Waldauer, S. A., Bakajin, O. \& Lapidus, L. J. Extremely slow intramolecular diffusion in unfolded protein L. Proc. Natl. Acad. Sci. 107, 13713-13717 (2010).

30. Soranno, A. et al. Quantifying internal friction in unfolded and intrinsically disordered proteins with single-molecule spectroscopy. Proc. Natl. Acad. Sci. 109, 17800-17806 (2012).

31. Sherman, E. \& Haran, G. Coil-globule transition in the denatured state of a small protein. Proc. Natl. Acad. Sci. 103, 11539-11543 (2006). 
32. Av, D. \& Jm, C. Swelling of Biological and Semiflexible Polyelectrolytes. Journal of physics. Condensed matter : an Institute of Physics journal vol. 21

https://pubmed.ncbi.nlm.nih.gov/21715847/ (2009).

33. Chen, H. et al. Ionic strength-dependent persistence lengths of single-stranded RNA and DNA. Proc. Natl. Acad. Sci. 109, 799-804 (2012).

34. D, C., N, H. \& D, T. Sequence-Dependent Three Interaction Site Model for Single- And Double-Stranded DNA. Journal of chemical theory and computation vol. 14 https://pubmed.ncbi.nlm.nih.gov/29870236/ (2018).

35. Grebikova, L. et al. The persistence length of adsorbed dendronized polymers. Nanoscale $\mathbf{8}$, 13498-13506 (2016).

36. S, G., L, S., N, D., M, M. \& C, T. Dependence of DNA Persistence Length on Ionic Strength and Ion Type. Physical review letters vol. 122 https://pubmed.ncbi.nlm.nih.gov/30720315/ (2019).

37. Skolnick, J. \& Fixman, M. Electrostatic Persistence Length of a Wormlike Polyelectrolyte. Macromolecules 10, 944-948 (1977).

38. Odijk, T. Polyelectrolytes near the rod limit. J. Polym. Sci. Polym. Phys. Ed. 15, 477-483 (1977).

39. Jacobson, D. R., McIntosh, D. B., Stevens, M. J., Rubinstein, M. \& Saleh, O. A. Singlestranded nucleic acid elasticity arises from internal electrostatic tension. Proc. Natl. Acad. Sci. U. S. A. 114, 5095-5100 (2017).

40. Mantelli, S., Muller, P., Harlepp, S. \& Maaloum, M. Conformational analysis and estimation of the persistence length of DNA using atomic force microscopy in solution. Soft Matter 7, 3412-3416 (2011).

41. Gittes, F., Mickey, B., Nettleton, J. \& Howard, J. Flexural rigidity of microtubules and actin filaments measured from thermal fluctuations in shape. J. Cell Biol. 120, 923-934 (1993).

42. Wisanpitayakorn, P., Mickolajczyk, K. J., Hancock, W. O., Vidali, L. \& Tüzel, E. Measurement of the Persistence Length of Cytoskeletal Filaments using Curvature Distributions. http://biorxiv.org/lookup/doi/10.1101/252551 (2018) doi:10.1101/252551.

43. Chin, A. F., Toptygin, D., Elam, W. A., Schrank, T. P. \& Hilser, V. J. Phosphorylation Increases Persistence Length and End-to-End Distance of a Segment of Tau Protein. Biophys. J. 110, 362-371 (2016). 